\title{
About the base problems of the geomechanics of strong compressed rock and rock masses
}

\author{
Vladimir Makarov ${ }^{* 1}$, Mikhail Guzev ${ }^{2}$ and Vladimir Odintsev ${ }^{3}$ \\ ${ }^{1}$ Far Eastern Federal University, 690014 Vladivostok, Russia \\ 2 Institute of Applied Mathematics FEB RAS, 690000 Vladivostok, Russia \\ ${ }^{3}$ Institute for the Problems of Integrated Development of Georesources RAS, 111020 Moscow, \\ Russia
}

\begin{abstract}
Some approaches to the simulation of hierarchically-block geomedia are considered. The idea of mesomechanics with allocation of mesovolume of representative scale level is used. It is offered to describe within the frame of geomechanics a rock mass four scale levels considering the conforming hierarchy of structural blocks. On each of these levels the rock mass is presented by non-Euclidian model of a continuous medium with the cracking defects, thus models of different scale levels differ only with values of parametres entering into them.
\end{abstract}

Key-words: geomedia, hierarchically-block rock mass, rock mass, structural levels, non-Euclidian model.

\section{Introduction}

Hierarchically-block character of a constitution of geomedium is recognized now by the majority of researchers, and search of adequate mathematical models of such medium is the actual problem demanding the prompt decision. In this direction, it is possible to emphasize two basic approaches, caused by the proposed principles of modelling.

First, it is a principle of construction of a continuous medium with inhomogeneities where the hierarchically-block geomedium is considered as an equivalent of continuous one, which properties are defined because of averaging on volume and with selection of a comprehensible range of the inhomogeneities $[1,2]$. A lack of such approach is, on the one hand, an arbitrariness in a choice of scales of averaging which results in set of various models, and, on the other hand, at fixing any "an averaging step" - appearance of set of the models which parametres definition is a formidable problem. Besides, as basic difficulty is "disappearing" of mesostructures from models because of the averaging operation which description of properties was supposed to be reached as a result of modelling.

Secondly, it is necessary to mention attempts of direct calculation of block system motion for different sizes where blocks are presented by rigid elements, and borders of blocks are presented by the deformable layers-linings replaced by rheological elements in the model. Reduction of such models to continuous ones demand introduction of simplifications, which are analogous for similar operations in a discrete case. As a result, we obtain the quality estimations having mainly theoretical interest [3, 4].

\footnotetext{
*Corresponding author: vlmvv@mail.ru
} 
Appearance of mesomechanical representations about scales and structural levels of destruction [5] seemed to be has approached the decision of a problem of the geomedium adequate model construction considering as discrete as continual character of its properties $[6,7]$. However the difficulties linked with the effective division of scale and structural levels of such medium, have not been overcame that has invoked representation about the mesomechanical approach, as deadlock with reference to construction of efficient model of geomedium.

Meanwhile, mesomechanics approaches to principles of the models of continuous mediums with defects construction have appeared rather effective with reference to plastic deforming and destruction of such materials, as metals and polymers [8]. The field method used here led directly to models, capable adequately to describe the mesostructures of "contrast" type, well known both at level of rock samples, and in a rock mass $[6,9,10]$.

In the present work authors, leaning against results of years of own study, represent the experimental establishments and the theoretical principles leading to necessity of introduction of non-Euclidian model of a continuous medium, combined both approaches of researches of hierarchically-block rock masses, and mesomechanics achievements with reference to building of integrative a mathematical model of geomedium.

\section{Short critical review of the modelling methods of modern geomechanics}

\subsection{The Principle of construction of a continuous medium with inhomogeneities where the hierarchically-block geomedium is changed by equivalent continuous one}

The block constitution of a rock mass has been found since the first works on discussion of an origin problem of tensile cracks in the conditions of all-round compression [11, 12]. Separation of rock mechanics in independent discipline, which a subject and methods essentially differ from methods of mechanics of soils, just has been caused by basic distinction of properties of block and continuous mediums [13].

Development of rock mechanics has led to understanding of a hierarchically-block constitution of a rock mass that has defined necessity of selection of hierarchy of blocks, where as criterion of reference to this or that level ability of rocks to destruction of certain type served. The first classification has been yielded in M.A. Sadovsky's works [1, 14] which, having considered physical objects from the size of atom, to planetary, has evolved the scale multiplier 3 defining the attitude of the size of blocks of the next levels of the general hierarchy.

In this case in the continuous mechanic as a result of averaging substantially inhomogeneous medium is replaced with some fictitious continuum possessing thus the same reactions to external forces, as well as that medium with which it replaces [1] for relations of lengths of defects

$$
\lambda>1>\mathrm{L}
$$

where $\lambda, \mathrm{L}$ - top and bottom border of a selected hierarchical level, accordingly; 1 - the average typical size for the selected hierarchical level of cracking defects.

The similar approach is sustained in V.N. Nikolaevsky's who has considered procedure of medium with cracking defects change to continuous one [2]. For a condition (1) the equations of mass balance, an impulse and quantity of motion for elementary volume $\Delta \mathrm{V}$ can be considered differential with intrinsic scale $\lambda$ (the size of a crystallite of grain, a pore etc.). 
In work [15] after introduction of «a fundamental initial series of geoblocks» (natural separatenesses), associated with a liquid core of the Earth in diameter of $2500 \mathrm{~km}$, objects of an initial row of geoblocks $\Delta_{i}$ (from planetary to fine-crystalline level) are defined by a range:

$$
\Delta_{i}=\Delta_{0} \cdot(\sqrt{2})^{i}, \Delta_{0} \approx 2,5 \cdot 10^{6} \mathrm{~m},
$$

where $i-$ the integers which are taking over negative values $(i=-1,-2,-3, \ldots)$ at transferring from large representatives of geostructure to smaller; ${ }^{\Delta}{ }^{-}$- diameter of a "liquid" core of the Earth.

It is noticed that in (2) at quantitative level the phenomenological model of a structurally-hierarchical constitution of geomedium is displayed at its partition from the big sizes to the smaller ones. Expression (2) leads to a range [9]:

$$
d_{j}=d_{0}(\sqrt{2})^{j-1} \approx d_{0} \frac{\sqrt{2}}{2} \exp (0,34466 j), j=1,2, \ldots, n,
$$

where ${ }^{d_{j}-}$ the representative size of natural separateness of a geomedia on $j-{ }_{\text {level of }}$ hierarchy; $d_{0}-$ the basic (minimum) size of natural separateness $\left(d_{0}=0,001 \mathrm{~mm}\right.$, $j=1,2, \ldots, 44$. ).

As follows from (3), at specified in [15] approach 44 models for the description of hierarchically-block geomedium are necessary that in engineering appendices leads to formidable problems.

\subsection{Methods of direct calculation of a block system movement of all possible sizes}

One-dimensional mathematical models of viscoelastic deforming of block mediums are considered in work [16] where it is shown that representation of blocks by massive rigid bodies with elastic layers between blocks gives the chance to describe precisely enough low-frequency slow waves (a wave of pendular type ), arising at shock influence.

In other work [4] energy diffusion to the block medium modelled by an onedimensional chain of masses, connected by springs and dampers is studied. Influence of parametres of block medium on an energy dissipation is investigated. The approximate analytical solution describing a total energy of block medium at wide interval of time is received.

It is necessary to mention also work [17] in which propagation of surface «pendular» waves at non-stationary influence on a free surface of the hole placed in block semispace is numerically investigated. A mathematical model of non-stationary viscoelastic deforming of the block medium is proposed. The model is based on representation that the dynamic behaviour of such medium can be approximately described as movement of rigid blocks because of a pliability of layers between them. For the description of viscoelastic behaviour of interblock layers the model of internal friction with a stuff Q-factor, as in the defining parameter is used. The medium is modelled by a three-dimensional lattice of the masses connected by elastic springs and viscous dampers in axial and diagonal directions. In the framework of this model the problem about non-stationary influence of type of the «dilating centre» on a hole surface, deepen in block semispace is numerically solved.

Because of numerical calculation it is shown that the basic contribution to wave process on a surface of block medium lead low-frequency longitudinal and Rayleigh "pendular" 
waves. Depending on size of deepening of a hole and distance from a place of influence the amplitudes of radial and vertical speeds of blocks in a longitudinal wave can be both above, and more low, than in Rayleigh wave.

In the considered work [17] it is underlined also «obvious limitation» to the model for description of real block mediums. As a whole, the specified approach allows to explain at qualitative level the effects linked with wave anomalies, caused by dynamic influences of mass explosions during mining processes. However, main process of the mesocracking structures forming in block medium they do not mention as the basic process of formation and the problem of block hierarchy is not considered also.

Even more mathematical model is offered by authors of work [18]. In their opinion, now «theory of block structures is constructed» as a result of working out by authors of a new method - «a differential method of factorization» which has demanded «attraction of the big panel of modern methods of mathematics not applied together ... the external analysis, methods of topology, the theory of functions of many complex variables, formresidues of Leray, the theory of group representation». Nevertheless, it has appeared insufficient, and it was necessary to add a new and not less powerful method «an integrated method of factorization». Such theory has allowed to create a new numerical method - a method of «a block element» which considerably differs from well-known methods of final and boundary elements. Problems of construction of mathematical models «for an intensity stressed lithosphere plates, in materials technology, building, nanotechnology» are as a result solved.

\subsection{Mesomechanics approach to principles of continuous media models with defects construction}

Appearance of «Physical mesomechanics» as the scientific discipline studying and modelling mesolevel of medium with defects has forced to scrap approaches to construction of adequate mathematical models of geomedium. Application of mesomechanics principles in geomechanics is reflected in P. V. Makarov and S. V. Goldin's works, where the basic approaches and modelling terminology with reference to geomedium have been formulated. Authors are guided by on such position of «Physical mesomechanics»: «Physical mesomechanics» considers a deformable stuff, as the organized system of structural levels of various scales. For the adequate description of processes of deformation and destruction, in accordance with the approach of physical mesomechanics, it is necessary simultaneous and inter consistent consideration of micro - meso- and macro - scales for which essentially different methods of modelling» should be elaborated [19].

Therefore working out of models of geomedium is represented to them as selection of «micro - meso- and macro - scales» in geomedium and division into these scales of structural elements of medium - on «structural levels», compounding hierarchy on each of scales.

Let's notice that the geomedium, since M. A. Sadovskii's classical works, represents a hierarchically-block rock mass [14]. The block structure has, as a rule, the form of "masonry" and is formed as a result of geological processes [13], and the size of blocks varies from $0,1 \mathrm{~mm}$ to first hundreds kilometers.

In work [7] it is taken into consideration the concept of divisibility of rocks on structural blocks for which there is a scale multiplier $\left\langle\Phi^{2}\right\rangle\left(\Phi^{2}=2,618\right.$ - one of Fibonacci numbers $)$ (instead of the value 3 proposed in work [1]) is introduced, in which connection «block» in [7] is considered as «dissipation structure». Such approach forces authors to find some classes of the nonlinear models which decision in the near future is represented as hardly feasible [7]. All it creates sensation of the deadlock for the scientific direction that has once again appeared powerless before complexity of such object, as geomedium at the 
researcher. Meanwhile, to such conclusion pushes not that other, as a position of authors of the considered above concept basing the conclusions, mainly, on experiments with metals.

\section{Principles of hierarchically block geomedium modelling}

\subsection{Selection of scale levels of hierarchically-block geomedium}

Really, what structural elements results S.V.Goldin results in his article, naming it «Destruction of lithosphere and the physical mesomechanics» [20]? Noticing that the geomedium is hierarchically-blocky, he, at transferring to mesolevel, formulates: «Basic types of mesoelements: strips of plastic flow and rather rigid domains .... The main types of deformations at mesolevel is a shear and rotation ...».

However, in geomechanics it well known that the basic structural element of geomedium is a crack. Moreover, it is the crack of shear-tensile type [21]. Crack can evolve on three scales: micro - meso- and macro- ones. For example, microcracks of the rock sample have intra crystalline (intra-grain) character, mesocracks evolve, as a rule, on borders of grains (diameter of grain $\boldsymbol{d}$ ) and have length from $\boldsymbol{d}$ to $(5-10) \boldsymbol{d}$ [22]. The macrocrack is a result of integration of mesocracks, and under the conditions of compression it is legibly possible to point out a stage of a sustainable development of a macrocrack and to find limiting length of steady macrodefect (Fig. 1).

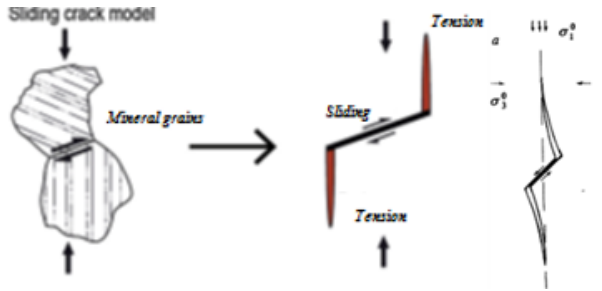

a)

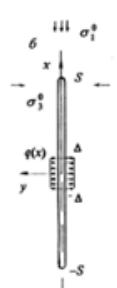

b)

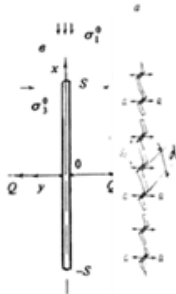

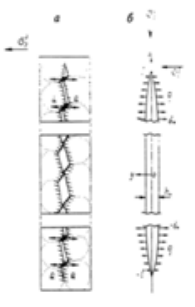

c)

Fig. 1. Stages of formation shear-tensile macrocracks and its modelling

Process of affiliation of mesocracks with macrodefect formation has the specificity: it is preceded by localisation of cracking process with formation source areas where, at stability loss, macrocrack growth descends jump. The majority of models of the locus of earthquakes is based on this process $[23,24]$. All these stages are well investigated on samples of rocks at monaxonic and volume loading [25]. Thus, mineral grains for the rock sample can be considered as blocks (Fig. 2).

At transferring to a rock mass we as find its block constitution already at level of the dimensions of chinks and mining openings. And blocks form a configuration which as it was specified above, in geomechanics it is accepted to name "masonry".

Repetition of the processes similar to the phenomena of destruction, positioned for rock samples, and unlimited growth of the macrocracks which have lost stability leads to formation in a rock mass of system of blocks. As a result the second hierarchical level of block structure of geomedium - the first structural level of a rock mass is formed. 


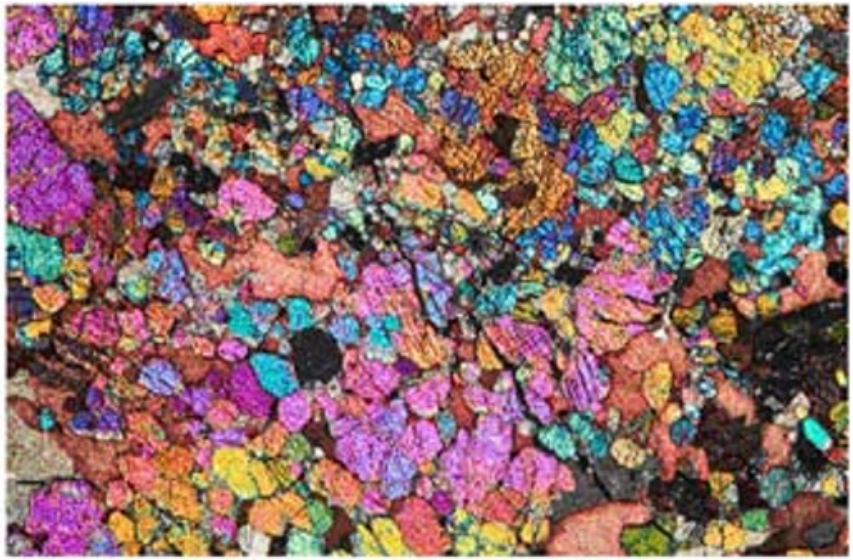

a)

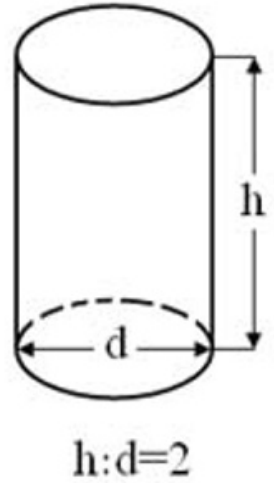

b)

Fig. 2. The first level of block hierarchy of geomedium: a - mineral grains in the sample of a granite; $\mathrm{b}$ - the schema of a cylindrical of rock sample

At this level the macrocrack of the sample acts in a role of a mesocrack of a rock mass (on borders of blocks), and mesocracks of the sample act as a microcrack (intrablock) of a rock mass.

In the conditions of the big compressing stresses in rock media, on borders of a rock mass blocks there is a formation of conditions of a rupture at compression again. Considered above for the rock sample process of mesodefects accumulation, their localisation in source areas with approach of the moment of interaction is retried. That is conducting to formation of a macrocrack and the second hierarchical level of blocks of a rock mass (it already third hierarchical level of block geomedium where the level of the rock sample considered above was the first one). The macrocrack of the second hierarchical level of the rock mass blocks, in turn, is a mesocrack at a following hierarchical level of geomedium etc.

The considered approach essentially wide the ideas of "Mesomechanics" with reference to hierarchically-block geomedium: the size of steady shear-tensile macrocracks defines the minimum size of the block of neighbor-top level and, accordingly, limits "from below" its level in block hierarchy. Thus the interblock shear-tensile cracks of the neighbor-low hierarchical level are considered how mesocracks, and intrablock - as microcracks.

Thus, at transferring to the next in hierarchy top structural level of blocks a macrocrack of the neighbor-low level are considered as a mesocrack of this neighbor-top level, and a mesocrack of the neighbor-low level - accordingly, as neighbor-top level microcracks. It is as a result had structural hierarchy of the geoblocks, each which structural level, since following the rock sample a rock mass level (modularity of the sample is defined by the natural size of minerals composing it), it is certain in the limiting sizes of steady macrodefect. In addition, at each structural level of geoblocks precipitate out micro-mesomacro- the scales directly bound to scales as neighbor-low as the top next level.

From this point of view, the block constitution, is perceived by us in the prograduated sense with reference to geomedium, since the rock sample, in comparison with traditional understanding $[1,15]$ or introduced in [7].

Other not less important element of construction of adequate model of geomedium is definition of mesovolume and on this basis - allocation of "representative" mesovolume [7]. 


\subsection{Selection of mesovolume from positions of physically reasonable sizing of the structural block}

In the considered above researches [7] working out of models of a hierarchically-block rock mass contacts with concept of mesovolume which presence of "all significant elements of a meso-constitution» is meant. The condition of strong compression imposes also restriction on properties of "representative" mesovolume: it is that minimum part of mesovolume, which is capable to reflect its properties under condition of interaction of mesodefects.

We saw above that interaction of shear-tensile mesocracks leads to their localisation with formation of source area of a macrocrack preparation. Not less important and that as show researches of near-source area, "reversive" character of deformations [27] here is found.

Reversive deforming indicates of local reduction of strains in the near-source areas while in source area the strain continue to increase. As a completely set source and nearsource areas represents mesocracking structure of «contrast type» on terminology [6].

Therefore, all fields of a highly compressed stuff of rock or a rock mass, where there is an interaction of mesocracks, it is possible to consider as mesovolume. From these positions, it is possible to consider as representative mesovolume that quantity of blocks of the conforming structural level with the interacting mesocracks educed on their borders, which is statistically significant from modelling positions.

Generally, for the rocks the size corresponding to the sizes of 30 mineral grains [28] that represents the sample which size oversteps the bounds of concepts «small» and where normal distribution of properties of grains is prevailing was taken over. The same approach could be conserved in case of the highly compressed rock sample. For a rock mass representative it would be possible to consider volume of 30 rock blocks of the conforming level in structural hierarchy with the mesocracks educed on their borders under condition of interaction between them.

The moment of the beginning of interaction of mesocracks directly precedes a point of reversal of linear strains in near-source area of the rock sample or a rock mass. For these experimentally fixed, conditions values of the module of deformation, lateral expansion coefficient and other parametres of representative mesovolume necessary for construction of model are defined.

Let us consider realization of the offered approach on an example of the two first hierarchical levels of block geomedium: the rock sample and a rock mass round underground openings.

\section{Hierarchically-block geomedium: structural levels}

As it is already considered above, in 1991 Lockner and Kuksenko have conducted research of laws of formation and development of macrocracks in rock samples with application of the newest servocontrolled technics for the process of destruction [25]. After a stage of chaotic cracking, there is a localisation of mesocracks and, after integration, they sprout in rupture.

Such models of macrocracks development were considered by one of the authors in 1996 in the monography where presence of a stage of a sustainable development of macrodefect has been proved under the specified experimental conditions of LocknerKuksenko [21]. 


\subsection{Definition of limiting steady length of shear-tensile rock macrocracks}

On borders of mineral grains as the most relaxed fields of a geomedia, at compression there are the shift mesodefects generating the tension fields in end section (see fig. 1). Sheartensile mesocracks combine with load growth, and in the sample the rupture macrocrack appears. The direction of its growth coincides with a direction of the maximum stress at axial compression of the sample. The macrocrack is modelled by a cut with the holding apart load distributed on the cut coasts. Expression of stress intensity factor for it looks like [21]:

$$
K_{1}=(\pi l)^{1 / 2}\left(\gamma_{1} \sigma_{1}^{0}-\gamma_{3} \sigma_{3}^{0}\right)
$$

where $\boldsymbol{I}$ - semilength of a crack, $\mathrm{m} ; \sigma^{\mathbf{0}}, \sigma^{0}{ }_{3}$ - accordingly the maximum and minimum principal stresses; $\boldsymbol{\gamma}_{1}, \boldsymbol{\gamma}_{\mathbf{3}}$ - empirical coefficients.

Presented in [21] theory of shear-tensile destruction of rocks describes all basic experimental facts known from tests of rock samples and allows to define the maximum length steady shear-tensile macrocracks. At the first hierarchical level of the block geomedium presented by rock samples (diameter of $3-5 \mathrm{~cm}$, height of $6-10 \mathrm{~cm}$ ), act as structural blocks the mineral grains having borders accurately precipitating out in experiment which set heterogeneity of the strength properties of rock of this hierarchical level. The attitude of the sizes of such blocks (diameter of mineral grain $0,1-0,5 \mathrm{~mm}$ ) to the size of the sample on the average compounds 1:100.

The sizes of microcracks for a mineral compound size of order of the mineral grain sizes [22]. The sizes of tensile mesocracks are arising at compression of the rock sample, variate in range from 1 until 5-10 diameters of grain [21]:

$$
\begin{gathered}
2 l_{\text {sam }}^{\text {meso-min }}=d_{\text {grain }}=(0,1 \div 0,5) \mathrm{mm} \\
2 l_{\text {sam }}^{\text {meso-max }}=(5 \div 10) d_{\text {grain }} .
\end{gathered}
$$

The sizes of critical length of macrocracks at axial compression $2 l_{* \text { sam }}^{\text {macro-max }}$ (limiting length of steady macrodefect) can be received from a following formula [21]:

$$
l_{*_{s a m}}^{\text {macro-max }}=\frac{h_{*} E}{4\left(1-v^{2}\right) \gamma_{1} \sigma_{C}}
$$

where $h_{*} \approx d_{\max }-$ size of the block in the laboratory sample; $E$ - rock elastic modulus; $\sigma_{c}-$ ultimate strength on axial compression; $v$ - rock Poisson's ratio; $d_{\max }-$ maximum diameter of mineral grain.

At transferring to a following top structural level in hierarchy of rock blocks of geomedium for openings in diameter of 3-5 $\mathrm{m}$ - that corresponds to conditions of the majority of the solitary mining openings of coal and ore treating industry - the interrelation 1:100 between the size of the rock sample and diameter of opening also is conserved.

If to believe that the principle of geometrical similarity between block structure of the of rock sample and a rock mass at hierarchically second level of block geomedium consideration of blocks of a rock mass demands that their sizes corresponded with the sizes of blocks of the inferior level as 1:100 [29] is fair. To them there correspond blocks $d_{\text {mas }}=(1 \div 5) \mathrm{cm}$, and the conforming sizes of mesodefects are defined as: 


$$
\begin{aligned}
& 2 l_{\text {mas }}^{\text {meso-min }}=d_{\text {mas }}=(1 \div 5) \mathrm{cm}, \\
& 2 l_{\text {mas }}^{\text {meso-max }}=(5 \div 10) d_{\text {mas }}
\end{aligned}
$$

It is easy to notice that on the average $2 l_{\text {mas }}^{\text {maso-min }}=d_{\text {mas }}=l_{* \text { sam }}^{\text {macro-max }}$, taking into account the form of blocks of a rock mass of type of «masonry» that can be accepted as an assessment at carrying out of tests for axial compression. It allows to consider macrodefect at hierarchically first level of the rock sample as mesodefect of the minimum length at hierarchically second (top) level of geomedium - level of mining opening. In this case the maximum length of a mesocrack of a rock mass will compound $2 l_{\text {mas }}^{\text {meso-max }} \approx(5 \div 10) \llbracket 2 l_{* \text { sam }}^{\text {macro-max }}=$ of a rock mass cracking systems [30]. We will notice also that application of expression (5) is hereinafter manufactured at, in whole, little change of the attitude $E / \sigma_{C} \approx$ const for various levels of geomedium [31].

\subsection{Sizes of shear-tensile cracks, faults of geomedium and the basic levels of block hierarchy}

Summing up to sizing of mesocracks at various levels of geomedium, it is possible to notice that as a mesocrack of next top in relation to the rock sample of hierarchical level of underground opening the steady macrocrack of the maximum testing length - the next inferior hierarchical level (Table 1) will serve.

Thus, interrelation requirements 1:100 are yielded by rock mass scale in which limens conditions of geometrical similarity [29] are conserved, and at conservation of mechanisms of destruction of rocks in a rock mass by rupture in the conditions of compression for openings in diameter to 3-5 m are carried out, except that, the requirement of physical similarity. Manufacturing, further, conduction of the received results on top levels of a rock mass within a crust, it is possible to receive assessments of the maximum sizes of steady macro-ruptures. Total generalizations are resulted in Table 2.

Taking into account linear approach of the theory, the settlement values resulted in Table 2, it is possible to consider satisfactory. Apparently, from the Table 2, all 4 hierarchical levels of blocks within a crust are evolved that does a problem of working out of the general model quite foreseeable.

After scaling in which frame the requirements of works $[1,2,7]$ are fulfilled on averaging of mesodefects, construction of model of a continuous medium with cracking defects at all levels of hierarchically-block rock mass goes on a constructive path because the shear a component at shear-tensile destruction remains defining in processes of formation of a macrocrack.

When comply with certain principles [33], it seems reasonable to apply mesomechanics models [7] to the geomedium at all levels of its block hierarchy. 
Table 1. Interrelation of the sizes of defects at various hierarchical levels of block structure of rocks.

\begin{tabular}{|c|c|c|c|c|}
\hline \multicolumn{2}{|c|}{ Level } & \multirow[t]{2}{*}{ Block Size } & \multicolumn{2}{|c|}{ Length of a crack } \\
\hline Hierarchical & Hierarchical & & Min & Max \\
\hline \multirow{2}{*}{ Sample } & Meso & $\begin{array}{l}d_{\max }- \\
\text { mineral grain size }\end{array}$ & $2 l_{\text {sam }}^{\text {meso-min }}=d_{\max }$ & $\begin{array}{l}2 l_{\text {sam }}^{\text {meso-max }}= \\
=(5 \div 10) d_{\max }\end{array}$ \\
\hline & Macro & $\begin{array}{c}d=30 \div 50 \mathrm{~mm} \\
- \text { sample } \\
\text { diameter } h=60 \div \\
100 \mathrm{~mm} \\
\text { - sample height }\end{array}$ & $\begin{array}{l}2 l_{\text {sam }}^{\text {macro-min }}= \\
=(5 \div 10) d_{\max }\end{array}$ & $\begin{array}{l}\frac{2 l_{*_{\text {sam }}^{\text {macro-max }}}^{\text {max }}=}{2\left(1-v^{2}\right) \gamma_{1} \cdot \sigma_{C}}\end{array}$ \\
\hline \multirow[b]{2}{*}{ Mass } & Meso & $\begin{array}{l}\text { Rock block size } \\
d_{\text {mas }}=l_{* \text { sam }}^{\text {macro }}\end{array}$ & $\begin{array}{l}2 l_{\text {mas }}^{\text {meso-min }}=d_{\text {mas }}= \\
=l_{*_{\text {sam }}}^{\text {macro-max }}\end{array}$ & $\begin{array}{l}2 l_{\text {mas }}^{\text {meso-max }}= \\
=(5 \div 10) d_{\text {mas }}\end{array}$ \\
\hline & Macro & $\begin{array}{l}\text { Size is equal to } \\
\text { diameter of } \\
\text { opening } \\
d_{o p} \div 10 d_{o p}\end{array}$ & $\begin{array}{l}2 l_{\text {mas }}^{\text {macro-min }}= \\
=(5 \div 10) d_{\text {mas }}\end{array}$ & $\begin{array}{c}2 l_{* \text { mas }}^{\text {macro-max }}= \\
\frac{h_{*}^{\text {mas }} \cdot E}{2\left(1-v^{2}\right) \gamma_{1} \sigma_{\text {res }}}\end{array}$ \\
\hline
\end{tabular}

Table 2. Structural blocks sizes at various hierarchical levels of geomedium

\begin{tabular}{|c|c|c|c|c|}
\hline Hierarchical level & $\begin{array}{c}\text { Structural } \\
\text { block size }\end{array}$ & $\begin{array}{c}\text { Macrodefect } \\
\text { steady length } \\
\text { size by } \\
\text { formula (5) }\end{array}$ & $\begin{array}{c}\text { Actual range } \\
\text { (structural block) }\end{array}$ & Citations \\
\hline Rock Sample & $0,5 \mathrm{~mm}$ & $10 \mathrm{~cm}$ & $0,1-0,5 \mathrm{~mm}$ & {$[21,22]$} \\
\hline $\begin{array}{c}\text { Rock Mass (mining } \\
\text { opening) }\end{array}$ & $5 \mathrm{~cm}$ & $10 \mathrm{~m}$ & $10-100 \mathrm{~cm}$ & {$[30]$} \\
\hline $\begin{array}{c}\text { Rock Mass } \\
\text { (excavation } \\
\text { opening) }\end{array}$ & $5 \mathrm{~m}$ & $1000 \mathrm{~m}$ & $2-5 \mathrm{~m}$ & {$[32]$} \\
\hline $\begin{array}{c}\text { Rock Mass (crust) } \\
\text { (n) }\end{array}$ & $500 \mathrm{~m}$ & $100 \mathrm{~km}$ & $10-230 \mathrm{~km}$ & $\begin{array}{c}{[1] \text { Lengths }} \\
\text { of breaks }\end{array}$ \\
\hline
\end{tabular}

The decisive factor here is the rejection of the kinematic conditions for the compatibility of Saint-Venant deformations [34] on the mesoscale, which leads to a transition from the classical elastic model of the medium to the non-Euclidean model. This allows us to formulate the principle of non-Euclidean hierarchy when building a model of a continuous 
medium with structural defects such as shear-tear-off cracks. It consists in the fact that the hierarchical-block array is replaced by a system of non-Euclidean models, each of which covers a particular structural level of the block hierarchy, allowing you to specify an adequate description of the mesocrack structures forming here. The principle of nonEuclidean hierarchy is supplemented by the «monolithic block» principle which means the consideration of intra-block defects as microcracks at each structural level. Wherein interacting mesocracks outlining the block correspond to the condition that in the general case there is no compatibility of deformations at each physical point represented by the block. Macrocracks at each structural level are single breaks without any interactions between them. Thus the contouring of block by interacting mesocracks answer an absence condition generally to compatibility of deformations in each physical point presented by the block. Macrocracks at each structural level are the solitary ruptures, interaction between which is absent.

With a set of such models and observing these principles, it becomes possible to determine the main characteristics of dissipative mesocrack structures of the geomedium, such as the sizes of the source and near source areas of mesostructures of the source type, the radial extent of zones in the case of zonal type mesostructures etc. at each of the considered structural levels.

\section{Conclusion}

In accordance with the formulated principle of non-Euclidean hierarchy, the modeling of the characteristics of dissipative mesocrack structures of rock samples and rock masses should be performed by calculation using non-Euclidean models of the corresponding hierarchical level. To this end, the following steps must be performed in sequence.

1. To establish a hierarchical structural level of a block geomedium on the basis of a geological assessment of the geoblocks size,

2. For the established structural level of the geomedium, determine the scale level of the (meso-macro) defective (fractured) structure,

3. To choose a non-Euclidean model of a continuous medium corresponding to the selected hierarchy level and the mesoscale defined for it,

4. To develop the methods for determining the phenomenological parameters of the non-Euclidean model of a continuous medium,

5. To solve boundary problems corresponding to the goals of modeling,

6. To develop algorithms and programs for calculating the parameters of dissipative mesocrack structures of the corresponding level of the block geomedium (rock samples or rock masses).

Thus, the general method for determining the characteristics of dissipative mesocrack structures of rock samples and rock masses is as follows. A non-Euclidean model of a continuous medium corresponding to the selected conditions is developed for a hierarchical level of a block geomedium, selected on the basis of a geological estimation of the geoblocks size, and a certain scale level of mesodefects (mesocracks). The mesostructure characteristics are calculated after solving the corresponding boundary value problems and determining the model parameters based on experimental data.

The research is supported by the Ministry of Science and High Education of the Russia Federation, unique identifier of the agreement RFMEFI58418X0034. 


\section{References}

1. M.A. Sadovsky, L.G. Bolhovitinov, V.F. Pisarenko, Deforming of geophysical medium and seismic process, Moscow: Science, (in Russian) (1987)

2. V. N. Nikolaevsky, Mechanics of fluid-saturated geomaterials: discusser's report, Wiley New York, 379-401 (1985)

3. V. M. Sadovskii, O. V. Sadovskaya, Wave Motion, 52, 138-150 (2015)

4. K. Vang, N. I. Aleksandrova, I. Pan, V. N. Oparin, L. Dow, A. I. Chanyshev, Applied mechanics and the technical physics, 60(5), 168-177 (in Russian) (2019)

5. The Physical mesomechanics and computer designing of stuffs: 2 volumes, Edt. V. E. Panin, Novosibirsk: Science, 1, 2, (in Russian) (1995)

6. S. V. Goldin, Physical mesomechanics, 8(1), 5-14 (in Russian) (2005)

7. P. V. Makarov, I. Yu. Smolin, Yu. P. Stefanov etc., The Nonlinear mechanics of geomaterials and geomediums, Edt. L. B. Zuev, (Novosibirsk: Geo, 2007) (in Russian)

8. V.E. Panin, Yu. V. Grinjaev, V. I. Danilov etc., Structural levels of a plastic strain and destruction, (Novosibirsk: Science, 1990) (in Russian)

9. Е. I. Шемякин, G. L. Fisenko, M. V. Kurlenja, V. N. Oparin etc., RAS USSR, 289(5), 1088-1094 (in Russian) (1986)

10. M. A. Guzev, V. V. Makarov, Deforming and destruction of highly compressed rocks and rock masses (Vladivostok: Dalnauka, 2007)

11. W. O. Crosby, Proc. Boston Society Natural History (Boston: Printed for the Society), 22, 72-85 (1884)

12. W. King, The Transactions of the Royal Irish Academy, 25, 605-662 (1875)

13. Rock mechanics, ed. by L. Müller, (Wien; New York: Springer, 1982)

14. M. A. Sadovsky, RAS USSR, 274(4) (1979)

15. V. N. Oparin, A. S. Tanajno, Classification, Physical-technical problems of working out of minerals, 6, 40- 53 (in Russian) (2009)

16. N. I. Aleksandrova, E. N. Sher, Modelling of process of wave propagation in block mediums, 6, 49-57 (in Russian) (2004)

17. N. I. Aleksandrova, Fundamental and applied questions of mining sciences, 6(1), 32-38 (in Russian) (2019)

18. V. A. Babeshko, O. M. Babeshko, O. V. Evdokimova, Ecological bulletin of the Black Sea economic cooperation, 1, 18-23 (in Russian) (2009)

19. P. V. Makarov, Approach of physical mesomechanics to modelling of processes of deformation and destruction, 1(1), 61-68 (in Russian) (1998)

20. S. V. Goldin, Physical mesomechanics, 5(5), 5-22 (2002)

21. V. N. Odintsev, Tensile destruction of a rock mass (Moscow: IPKON RAS, (in Russian) 1996)

22. A. N. Kochanov, Mining information-analytical bulletin (scientific and technical magazine), 7, 221-225 (in Russian) (2015)

23. J. R. Rice The mechanics of earthquake rupture, Physics of the Earth's Interior, (Italian Physical Society, 1980)

24. I. P. Dobrovolsky, Theory of preparation of tectonic earthquake, (Moscow: RAS, (in Russian) 1991)

25. D. A. Lockner, V. S. Kuksenko et al., Nature, 350(6313), 39-42 (1991) 
26. Procedure of drawing up of geoblock diagrams (models) of a rock masses in the establishments of hydroconstructions, Manual for Building regulations 2.02.02-85, Leningrad: VNIIG, (in Russian) (1991)

27. V. V. Makarov, L. S. Ksendzenko, A. M. Golosov \& N. A. Opanasiuk, Rock Engineering and Rock Mechanics: Structures in and on Rock Masses, Proceedings of EUROCK 2014, 267-272 (2014)

28. K. V. Ruppenejt, Yu. M. Liberman Introduction in Rocks Mechanics, Moscow: Gosgortehizdat, 43, 95-111 (in Russian) (1960)

29. L. I. Sedov, Similarity and Dimensional Methods in Mechanics, $10^{\text {th }}$ Edition, CRC Press (1993)

30. S. N. Chernyshev Discontinuities of rocks (Moscow: Nauka, (in Russian) 1983)

31. V. V. Makarov, M. A. Guzev, V. N. Odintsev, L. S. Ksendzenko, Journal of Rock Mechanics and Geotechnical Engineering, 8(2), 164-169 (2016)

32. V. P. Lushpej, V. V. Makarov, A. S. Laptev, Kolyma, 3-4, 18-21 (in Russian) (1982)

33. M. A. Guzev, V. N. Odintsev, V. V. Makarov, Tunnelling and Underground Space Technology, 81, 506-511 (2018)

34. C. Jaeger, N. G. W. Cook, R. W. Zimmerman Fundamentals of Rock Mechanics, Blackwell Publishing (2007) 